\title{
Experimental determination of electric cross-spectral density matrix and generalized Stokes parameters for a laser beam
}

\author{
Bhaskar Kanseri and Hem Chandra Kandpal* \\ Optical Radiation Standards, National Physical Laboratory, New Delhi 110012, India \\ *Corresponding author: hckandpal@mail.nplindia.ernet.in
}

Received May 29, 2008; revised July 13, 2008; accepted August 6, 2008; posted September 18, 2008 (Doc. ID 96820); published October 15, 2008

\begin{abstract}
We report an experimental method to determine the elements of the electric cross-spectral density matrix for laser light. For this purpose an additional setup consisting of mirrors and reflecting prisms is utilized with the conventional Young's interferometer to overcome existing experimental limitations. The generalized Stokes parameters, which are the characteristics of two spatial points of the electromagnetic field, are also obtained for a pair of points. The knowledge of these two quantities might be useful in determining the change in polarization properties of light in propagation and their effects in optical measurements. (C) 2008 Optical Society of America
\end{abstract}

OCIS codes: $260.3160,030.1640$.

The recently developed unified theory of coherence and polarization by Wolf [1] suits well with the observed theoretical and experimental observations [2-4] that could not fully be explained by the Stokes parameters [5] or $2 \times 2$ coherence matrix [6]. The unified theory elucidates an intimate relationship existing between the coherence and the polarization of electromagnetic beams. The central quantity in this theory is a $2 \times 2$ electric cross-spectral density matrix $\overleftrightarrow{W}\left(\mathbf{r}_{1}, \mathbf{r}_{2}, \omega\right)$, which characterizes the correlations between two mutually orthogonal spectral components of the electric field at a pair of points in a plane perpendicular to the direction of propagation of the beam. Later, using the newly presented matrix, a lot of theoretical and experimental work has been done treating the light field as a vector quantity [7-9]. The recently introduced generalized Stokes parameters [10] also depend on two spatial variables. In a nutshell we can say that the generalized Stokes parameters, like the cross-spectral density matrix, could also be used to study both the coherence and the polarization properties of random electromagnetic beams.

After the invention of the laser, most of the optical experiments are conducted using a laser source. Thus it becomes of the utmost importance to have information about the polarization properties of the laser beam. In the present Letter, we have experimentally determined the cross-spectral density matrix of an expanded laser beam at a pair of points in the doubleslit plane. An expanded laser beam was used so that the secondary source (the pinhole of the beam expander) could approximately simulate the point source with reduced spatial coherence.

The method used here is an experimental realization of the theoretical approach presented in [11] to obtain the components of the cross-spectral density matrix using polarizers and rotators in front of the pinholes and taking spectral measurements at the observation plane. To our knowledge this approach [11] has yet not been realized experimentally prob- ably owing to the complexities involved in existing spatial coherence based interferometers for placing polarizers and rotators before the individual pinholes, which were having very narrow separation (typically few hundreds of micrometers). Introducing a combination of prisms and mirrors after the double slit, the two beams were separated by a few centimeters to overcome the prevailing complexities. The four generalized Stokes parameters that involve the same pair of points were also determined.

Let us consider a random electromagnetic beam propagating in the $z$ direction. The fluctuations of the beam could be considered statistically stationary at least in a wide sense. Let $E(\mathbf{r}, \omega)=\left\{E_{i}(\mathbf{r}, \omega)\right\},(i=x, y)$ be the statistical ensemble of frequency $\omega$ of the fluctuating electric field $E(\mathbf{r}, \omega)$ at a point $\mathbf{r}$ in space. The second-order coherence properties of the beam may be characterized by a $2 \times 2$ electric cross-spectral density matrix [1]:

$$
\begin{aligned}
\overleftrightarrow{W}\left(\mathbf{r}_{1}, \mathbf{r}_{2}, \omega\right)= & W_{i j}\left(\mathbf{r}_{1}, \mathbf{r}_{2}, \omega\right)=\left\langle E_{i}^{*}\left(\mathbf{r}_{1}, \omega\right) E_{j}\left(\mathbf{r}_{2}, \omega\right)\right\rangle, \\
& i=x, y, j=x, y,
\end{aligned}
$$

where the asterisk denotes the complex conjugate.

Let an opaque screen be placed across the plane $z$ $=0$ with two narrow slits symmetrically positioned about the line perpendicular to the plane (inset of Fig. 1). Let $Q_{1}$ and $Q_{2}$ be any two points having posi-

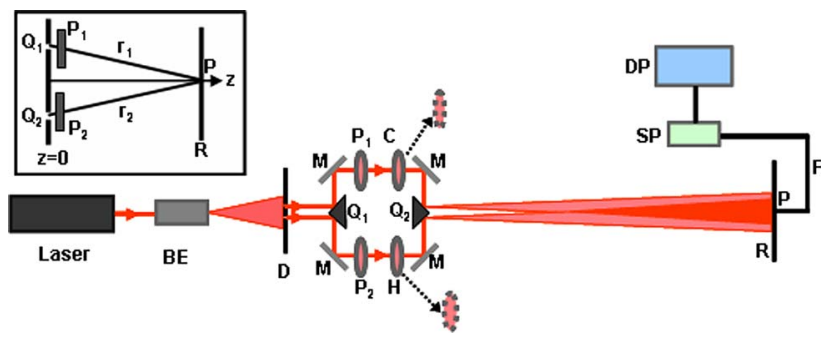

Fig. 1. (Color online) Schematics of the experimental setup. The inset illustrates the notations. Abbreviations are defined in the text. 
tion vectors $\mathbf{r}_{1}$ and $\mathbf{r}_{2}$, respectively, in the different slits. The spectral degree of coherence of the electric field of the beam is given by [4]

$$
\eta\left(\mathbf{r}_{1}, \mathbf{r}_{2}, \omega\right)=\frac{\operatorname{Tr} \overleftrightarrow{W}\left(\mathbf{r}_{1}, \mathbf{r}_{2}, \omega\right)}{\sqrt{\operatorname{Tr} \overleftrightarrow{W}\left(\mathbf{r}_{1}, \mathbf{r}_{1}, \omega\right)} \sqrt{\operatorname{Tr} \stackrel{\leftrightarrow}{W}\left(\mathbf{r}_{2}, \mathbf{r}_{2}, \omega\right)}} .
$$

Equation (2) can be expressed in the form [11]

$$
\operatorname{Tr} \overleftrightarrow{W}\left(\mathbf{r}_{1}, \mathbf{r}_{2}, \omega\right)=\sqrt{S\left(\mathbf{r}_{1}, \omega\right)} \sqrt{S\left(\mathbf{r}_{2}, \omega\right)} \eta\left(\mathbf{r}_{1}, \mathbf{r}_{2}, \omega\right) .
$$

According to [11], by using different polarizing elements before the separate slits, the components of the cross-spectral density matrix $\overleftrightarrow{W}\left(\mathbf{r}_{1}, \mathbf{r}_{2}, \omega\right)$ will be given by

$$
\begin{aligned}
\overleftrightarrow{W}\left(\mathbf{r}_{1}, \mathbf{r}_{2}, \omega\right)= & W_{i j}\left(\mathbf{r}_{1}, \mathbf{r}_{2}, \omega\right) \\
= & \sqrt{S_{i}\left(\mathbf{r}_{1}, \omega\right)} \sqrt{S_{j}\left(\mathbf{r}_{2}, \omega\right)} \eta_{i j}\left(\mathbf{r}_{1}, \mathbf{r}_{2}, \omega\right), \\
& i=x, y, j=x, y .
\end{aligned}
$$

The generalized Stokes parameters, which depend upon two spatial points, are different than the usual Stokes parameters, which are one-point quantities. The first parameter $S_{0}\left(\mathbf{r}_{1}, \mathbf{r}_{2}, \omega\right)$ gives the total intensity of the field at two points, while the second parameter $S_{1}\left(\mathbf{r}_{1}, \mathbf{r}_{2}, \omega\right)$ is the measure of the linear polarization in orthogonal $x$ and $y$ directions of the points. The third parameter $S_{2}\left(\mathbf{r}_{1}, \mathbf{r}_{2}, \omega\right)$ provides information about the linear polarization at $45^{\circ}$ of the $x$ and the $y$ axis at the two points, and the fourth parameter $S_{3}\left(\mathbf{r}_{1}, \mathbf{r}_{2}, \omega\right)$ is a measure of the circular polarization for the points. All the four generalized Stokes parameters could be expressed as [10]

$$
S_{i}\left(\mathbf{r}_{1}, \mathbf{r}_{2}, \omega\right)=\operatorname{Tr}\left[\overleftrightarrow{W}\left(\mathbf{r}_{1}, \mathbf{r}_{2}, \omega\right) \cdot \overleftrightarrow{\sigma}_{i}\right] \quad(i=0,1,2,3),
$$

where $\overleftrightarrow{\sigma}_{i}(i=1,2,3)$ are the Pauli spin matrices [6] and $\overleftrightarrow{\sigma}_{0}$ is the unit matrix.

A randomly polarized helium-neon laser (Melles Griot, wavelength $632.8 \mathrm{~nm}$, power $2 \mathrm{~mW}$ ) was used as a source (Fig. 1). The laser beam was expanded using a spatial filter beam expander assembly $(\mathrm{BE})$ and was passed through a double slit (D, slit width $150 \mu \mathrm{m}$ and slit separation $200 \mu \mathrm{m}$ ) put in the beam path at $30 \mathrm{~cm}$ away from the expander and was symmetric about the line passing through the pinhole of the $\mathrm{BE}$, as shown in Fig. 1. Two right-angle frontcoated prisms $\mathrm{Q}_{1}$ and $Q_{2}$ were introduced after $\mathrm{D}$, one of which $\left(Q_{1}\right)$ separated the two beams approximately by $8 \mathrm{~cm}$, and the other $\left(Q_{2}\right)$ recombined them again at a distance $10 \mathrm{~cm}$ (along the $z$ axis) from the first prism. Mirrors (M) were used with prisms to guide the beam through required path. The straightline interference fringes were obtained in the observation plane $R$ at a distance $135 \mathrm{~cm}$ from the second prism $Q_{2}$ and were photographed as shown in Fig. 2. A fiber (F)-coupled spectrometer (SP, Photon Control Model No. SPM-002) mounted with a computer (DP)controlled motorized micropositioner was used to measure the spectra at the observation plane.

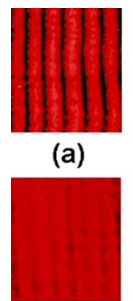

(c)

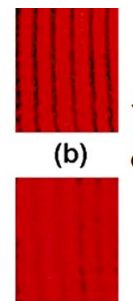

(d)

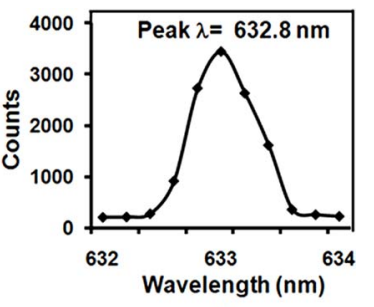

(e)
Fig. 2. (Color online) (a-d) Interference fringes observed at the observation plane $\mathrm{R}$ for different direction of polarizations (DOPs) of the polarizers. (e) Data recorded by the spectrometer shows the spectral profile of laser beam.

To calculate the $W_{x x}\left(\mathbf{r}_{1}, \mathbf{r}_{2}, \omega\right)$ component of the electric cross-spectral density matrix, two identical dichroic sheet polarizers (Melles Griot, wavelength range $380-780 \mathrm{~nm}$ ) having direction of polarization along the $x$ axis $\left(\mathrm{P}_{1}, \mathrm{P}_{2}\right)$ were inserted in the separate beam paths (Fig. 1). This allowed the $x$ components of the beams only to pass through, and good visibility interference fringes were obtained at $R$ as shown in Fig. 2(a). The maximum and minimum values of the spectral density were recorded by the spectrometer tracing the fiber tip horizontally over the central interference fringe. The data output of the spectrometer (saved in excel format) looks like Fig. 2(e). The spectral densities at an axial point $P$ over the central fringe due to the individual slits (points $Q_{1}$ and $Q_{2}$ ) were obtained by making measurements when only one slit was open and the other closed. To compute the $W_{y y}\left(\mathbf{r}_{1}, \mathbf{r}_{2}, \omega\right)$ component, both polarizers were rotated clockwise by $90^{\circ}$ (this made the direction of polarization $y$ axis), and similar measurements were made for both the slits which open simultaneously and alternately [fringes are shown in Fig. 2(b)].

The $W_{x y}\left(\mathbf{r}_{1}, \mathbf{r}_{2}, \omega\right)$ component of the matrix was obtained when the direction of polarization of the polarizer placed in one beam path was along the $x$ axis $\left(\mathrm{P}_{1}\right)$ while the other was along the $y$ axis $\left(\mathrm{P}_{2}\right)$. To bring the vibrations in the same plane so that interference could be possible, a half-wave plate $(\mathrm{H}$, multiorder, $\lambda=632.8 \mathrm{~nm}$ ), having optics axis at $45^{\circ}$ with the incident polarization of light was placed after the polarizer $\mathrm{P}_{2}$, which worked as a $90^{\circ}$ polarization rotator. Another half-wave plate $\mathrm{C}$ with identical specifications having optic axis along the incident polarization of light was placed in another arm for path compensation (Fig. 1). The maximum and minimum values of the spectral density for the same pair of points were recorded by the spectrometer at the observation plane for the central fringe [Fig. 2(c)]. With one slit closed and the other open, the spectral densities due to the individual slits were also recorded at the same point $\mathrm{P}$. The $W_{y x}\left(\mathbf{r}_{1}, \mathbf{r}_{2}, \omega\right)$ component was obtained by rotating both the polarizers by $90^{\circ}$ and taking similar spectral measurements for both the slits as well as the individual slits [fringes in Fig. 2(d)]. It is evident from the photographs that the fringe visibility due to $x y$ and $y x$ components is much less than the $x x$ and $y y$ components.

Following the procedure as stated earlier, the values of spectral densities about the central bright 
Table 1. Components of the Spectral Degree of Coherence and the Cross-Spectral Density Matrix Obtained for the Laser Light

\begin{tabular}{llll}
\hline$\eta_{x x}\left(\mathbf{r}_{1}, \mathbf{r}_{2}, \omega\right)$ & $0.84 \pm 0.09$ & $W_{x x}\left(\mathbf{r}_{1}, \mathbf{r}_{2}, \omega\right)$ & $410.6 \pm 4.0$ \\
$\eta_{x y}\left(\mathbf{r}_{1}, \mathbf{r}_{2}, \omega\right)$ & $0.32 \pm 0.06$ & $W_{x y}\left(\mathbf{r}_{1}, \mathbf{r}_{2}, \omega\right)$ & $118.2 \pm 1.6$ \\
$\eta_{y x}\left(\mathbf{r}_{1}, \mathbf{r}_{2}, \omega\right)$ & $0.24 \pm 0.04$ & $W_{y x}\left(\mathbf{r}_{1}, \mathbf{r}_{2}, \omega\right)$ & $119.7 \pm 1.8$ \\
$\eta_{y y}\left(\mathbf{r}_{1}, \mathbf{r}_{2}, \omega\right)$ & $0.71 \pm 0.08$ & $W_{y y}\left(\mathbf{r}_{1}, \mathbf{r}_{2}, \omega\right)$ & $366.4 \pm 3.4$ \\
\hline
\end{tabular}

Table 2. Generalized Stokes Parameters for a Pair of Points in the Laser Beam

$\begin{array}{cc}S_{0}\left(\mathbf{r}_{1}, \mathbf{r}_{2}, \omega\right) & 777.0 \pm 5.1 \\ S_{1}\left(\mathbf{r}_{1}, \mathbf{r}_{2}, \omega\right) & 44.2 \pm 2.5 \\ S_{2}\left(\mathbf{r}_{1}, \mathbf{r}_{2}, \omega\right) & 237.9 \pm 2.4 \\ S_{3}\left(\mathbf{r}_{1}, \mathbf{r}_{2}, \omega\right) & (1.5 \pm 0.81) i\end{array}$

fringe were measured at the observation plane $R$. The modulus of spectral degree of coherence of light (also called spectral visibility) for the two points was calculated using the formula [6]

$$
\frac{S_{\max }-S_{\min }}{S_{\max }+S_{\min }}
$$

where $S_{\max }$ and $S_{\min }$ are the respective maximum and the minimum values of spectral density around the central fringe when both the slits are open.

The values of the spectral degree of coherence of the electric field $\eta\left(\mathbf{r}_{1}, \mathbf{r}_{2}, \omega\right)$ for all the four cases are determined from the experimental data using the well-known methods [11] and are given in Table 1. In a similar manner, the spectral densities of the expanded laser beam due to the individual slits were measured at the axial point P. Using Eq. (4), we have calculated the elements of the electric cross-spectral density matrix as shown in Table 1. As the experiment was conducted eight times, the statistical error associated with it is shown as the standard deviation in the calculated values.

Using Eq. (4) the matrix would be given as

$$
\overleftrightarrow{W}\left(\mathbf{r}_{1}, \mathbf{r}_{2}, \omega\right)=\left[\begin{array}{ll}
410.6 \pm 4.0 & 118.2 \pm 1.6 \\
119.7 \pm 1.8 & 366.4 \pm 3.4
\end{array}\right] .
$$

Using the elements of the electric cross-spectral density matrix, the generalized Stokes parameters could be obtained using Eq. (5). The results are presented in Table 2.

Equation (7) shows that the spectral degree of coherence, i.e., visibility of interference fringes for cross-polarized cases ( $x y$ and $y x$ ) is less than that for the copolarized cases $(x x$ and $y y)$. It is due to the fact that there is no correlation (practically very small) between the two orthogonal components of the randomly polarized light [6]. Hence the condition for interference is satisfied only for the electric field, which is very little correlated [see Figs. 2(c) and 2(d)].

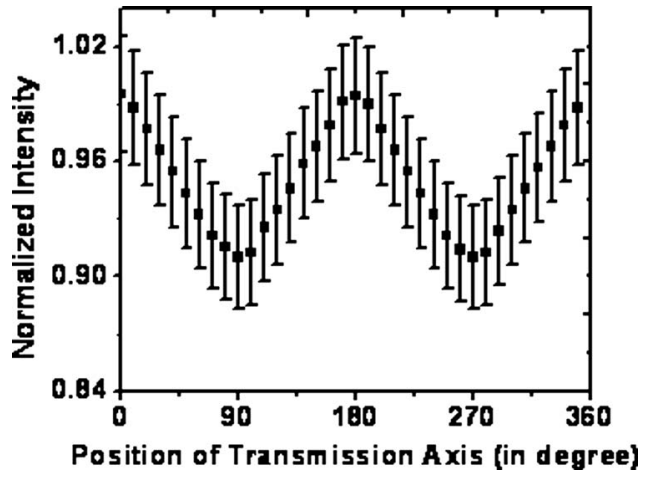

Fig. 3. Variation in the output intensity of the laser beam with different DOPs of the linear polarizer. $0^{\circ}$ and $180^{\circ}$ of the transmission axis correspond to the $x$ axis polarization.

It is evident from the positive value of the second Stokes parameter that for the two points, the laser beam has the $x$-axis polarization stronger than the $y$-axis polarization [Figs. 2(a) and 2(b)]. The same result could be observed (Fig. 3) by using a linear polarizer in front of the random laser beam and recording the output intensity as a function of the angular position of the transmission axis of the polarizer $\left(0^{\circ}\right.$ to $360^{\circ}$ ). Determining the Stokes parameters for different pairs of points in the propagating beam, the change in polarization owing to propagation could be measured.

In conclusion, in this study we have determined the four elements of the electric cross-spectral density matrix for a laser beam using a modified interferometer. The generalized Stokes parameters for a pair of points in the double-slit plane were also obtained using the density matrix. This simple method could be beneficial to study the properties of coherence and polarization of the random electromagnetic beams in propagation.

The authors thank the Director, National Physical Laboratory New Delhi for permission to publish the paper. B. Kanseri is grateful to the Council of Scientific and Industrial Research (CSIR) (India) for financial support as a Junior Research Fellowship (JRF).

\section{References}

1. E. Wolf, Phys. Lett. A 312, 263 (2003).

2. D. F. V. James, J. Opt. Soc. Am. A 11, 1641 (1994).

3. G. P. Agrawal and E. Wolf, J. Opt. Soc. Am. A 17, 2019 (2000).

4. E. Wolf, Opt. Lett. 28, 1078 (2003).

5. G. G. Stokes, Trans. Cambridge Philos. Soc. 9, 399 (1852).

6. L. Mandel and E. Wolf, Optical Coherence and Quantum Optics (Cambridge U. Press, 1995).

7. H. Roychowdhury and E. Wolf, Opt. Commun. 252, 268 (2005).

8. F. Gori, M. Santarsiero, R. Borghi, and E. Wolf, Opt. Lett. 31, 688 (2006).

9. E. Wolf, Introduction to Theory of Coherence and Polarization of Light (Cambridge U. Press, 2007).

10. O. Korotkova and E. Wolf, Opt. Lett. 30, 198 (2005).

11. H. Roychowdhury and E. Wolf, Opt. Commun. 226, 57 (2003). 